\title{
Linear Predictive Detection for Power Line Communications Impaired by Colored Noise
}

\author{
Riccardo Pighi and Riccardo Raheli \\ Dept. of Information Engineering, University of Parma, 43100 Parma, Italy \\ Email:pighi@tlc.unipr.it, raheli@unipr.it
}

\begin{abstract}
Robust detection algorithms capable of mitigating the effects of colored noise are of primary interest in communication systems operating on power line channels. In this paper, we present a sequence detection scheme based on linear prediction to be applied to single carrier power line communication impaired by colored Gaussian noise. The proposed solution improves the Bit Error Rate (BER) performance with respect to a conventional state-of-the-art system and makes the receiver more robust against colored noise. As a case study, we investigate the performance of the proposed receiver in a high-voltage power line channel limited by corona noise.
\end{abstract}

\section{INTRODUCTION}

Power line communication (PLC) channels provide a potentially convenient communication medium for control signaling and data communication. Even though power lines are an attractive and inexpensive solution for data transmissions, a reliable high-speed communication is a great challenge due to the nature of the medium. Any transmission scheme applied to power lines has to cope with several impairments such as frequency-varying and time-varying attenuation of the medium, intrinsic dependence of the channel model on the network topology and connected loads, the presence of high-level interference signals due to noisy loads and the presence of colored noise. Moreover, the power line channel is also limited by disturbances produced by events outside the transmission channel such as, for example, lightening [1] or disturbances originating within the system, such as network switching [2], impulse noise [3] and corona phenomena [4][6].

For narrowband applications in power lines, single carrier modulations may be adopted for their simplicity, based on quadrature amplitude modulation (QAM) or other modulation formats. However, in broadband applications strong colored noise sources can severely limit the performance of single carrier systems and demand for adequate signal processing schemes.

In this paper, we propose a single carrier PLC scheme based on linear prediction and multidimensional coding, which exhibits good improvements, in terms of bit error rate (BER) performance, with respect to a state-of-the-art industrial solution. The linear predictive approach is a valuable and general technique that can be used every time a communication system has to cope with colored noise [7], provided that a correct statistical information on the noise is available at the receiver. First, we will introduce the linear predictive detection scheme considering a general model for the colored noise process.

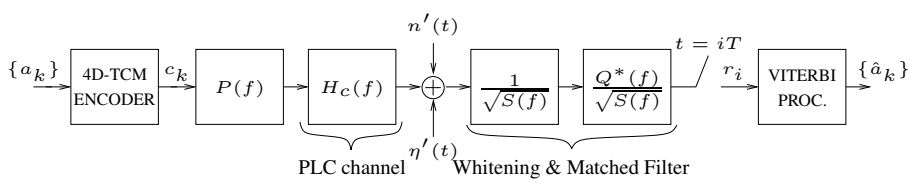

Fig. 1. Simplified system model with optimum receiver for colored noise.

As a case study, we will also analyze the performance of the proposed receiver considering corona noise [5], [6] as a particular type of colored noise. Moreover, in order to reduce the computational load of the linear predictive receiver, we apply reduced-state sequence detection techniques [8]-[11], such as "trellis folding by set partitioning" [12] and Per-Survivor Processing (PSP) [11], and demonstrate the robustness of the proposed scheme in terms of BER and complexity with respect to well-established industrial solutions.

\section{LINEAR PREDICTION RECEIVER}

Single carrier transmission may be attractive from a complexity point of view. However, since the power line channel is affected by severe intersymbol interference (ISI), powerful detection and equalization techniques are necessary. On the other hand, the practical implementation of these schemes also requires reduced-state approaches.

\section{A. Optimum Detector}

Let us consider the transmission scheme depicted in Fig. 1. We adopt a transmission system based on an eight-state fourdimensional trellis coded modulation scheme (4D-TCM) [13]. We assume a square-root raised cosine shaping filter with frequency response $P(f)$ and a power line channel with frequency response $H_{c}(f)$. The presence of colored noise with power spectral density given by $S(f)=S_{\eta^{\prime}}(f)+S_{n^{\prime}}(f)$, being $S_{\eta^{\prime}}(f)$ and $S_{n^{\prime}}(f)$ the power spectral densities of white thermal and colored noise, respectively, and the need for statistical sufficiency yield a detector front-end based on a whitening filter, with frequency response $1 / \sqrt{S(f)}$, and a filter matched to the overall channel response $Q^{*}(f) / \sqrt{S(f)}$, where $Q(f)=P(f) H_{c}(f)$ [14]. The resulting overall frontend filter $Q^{*}(f) / S(f)$ is a standard matched filter for colored noise [14]. We use a sequence detection Viterbi processor, searching an extended trellis diagram including ISI and the encoder memory, and using linear prediction to whiten the colored noise at its input. 
As a consequence, considering the system model in Fig. 1, the discrete time observable at the input of the Viterbi processor can be expressed as

$$
r_{i}=\underbrace{\sum_{n=0}^{L} g_{n} c_{i-n}}_{s_{i}\left(\boldsymbol{c}_{i-L}^{i}\right)}+\eta_{i}+n_{i}
$$

where $g_{i}=\left.g(t)\right|_{t=i T}=\left.p(t) * h_{c}(t) * m(-t)\right|_{t=i T}$ is the overall impulse response of the system with $m(t)=\mathcal{F}^{-1}\{M(f)\}$ and $M(f)=Q(f) / S(f), s_{i}\left(\boldsymbol{c}_{i-L}^{i}\right)$ is the noiseless signal component affected by ISI of length $L, \eta_{i}=\left.\eta^{\prime}(t) * m(-t)\right|_{t=i T}$ is a sample of Gaussian thermal noise and $n_{i}=\left.n^{\prime}(t) * m(-t)\right|_{t=i T}$ is a sample of a generic colored noise. We assume that the colored noise can be also modeled as a process with Gaussian statistics.

We now derive the optimal branch metric for a single carrier communication scheme to be used in a sequence detection Viterbi algorithm. Collecting the samples at the output of the colored noise channel into a suitable complex vector $\boldsymbol{r}$, we can formulate the Maximum A-Posteriori Probability (MAP) sequence detection strategy as

$$
\hat{\boldsymbol{a}}=\underset{\boldsymbol{a}}{\operatorname{argmax}} p(\boldsymbol{r} \mid \boldsymbol{a}) P\{\boldsymbol{a}\}
$$

where $p(\boldsymbol{r} \mid \boldsymbol{a})$ is the conditional probability density function (PDF) of the vector $\boldsymbol{r}$, given the data vector a and $P\{\boldsymbol{a}\}$ is the a priori probability of the information symbols. Since the trellis encoder can be described as a time-invariant finite state machine, it is possible to define a sequence of 4D states $\left\{\mu_{0}, \mu_{1}, \ldots\right\}$ over which the encoder evolves and define a deterministic state transition law, function of the 4D information symbol $a_{k}$, which describes the evolution of the system, i.e., $\mu_{k}=f\left(\mu_{k-1}, a_{k-1}\right)$. Note that each state $\mu_{k}$ belongs to a set of finite cardinality. As a consequence, the evolution of the finite state machine of the 4D-TCM encoder can be described through a trellis diagram, in which there is a fixed number of exiting branches from each state: this number will depend on the number of subsets in which the constellation is partitioned [15].

The 4D-TCM code symbol $C_{k}\left(a_{k}, \mu_{k}\right)=\left(c_{2 k-1}\left(a_{k}, \mu_{k}\right)\right.$, $\left.c_{2 k}\left(a_{k}, \mu_{k}\right)\right)$, with 2D components belonging to a QAM constellation, is a function of the encoder state $\mu_{k}$ and the information symbol $a_{k}$ at the input of the encoder. Note that $c_{2 k-1}\left(a_{k}, \mu_{k}\right)$ and $c_{2 k}\left(a_{k}, \mu_{k}\right)$ are, respectively, the first and second two dimensional (2D) symbol transmitted over the channel during the four-dimensional time interval. Under these assumptions, we can write the 4D discrete-time observable as $R_{k}=\left(r_{2 k-1}, r_{2 k}\right)$, where the 2D components are defined using (1).

Assuming causality and finite-memory [16], applying the chain factorization rule to the conditional PDF and taking into account the multidimensional structure of the TCM code, we can rewrite (2) as

$$
\hat{\boldsymbol{a}}=\underset{\boldsymbol{a}}{\operatorname{argmax}} \prod_{k=0}^{K-1} p\left(R_{k} \mid \boldsymbol{R}_{0}^{k-1}, \boldsymbol{a}_{0}^{k}\right) P\left\{a_{k}\right\}
$$

$$
\begin{array}{r}
\simeq \underset{\boldsymbol{a}}{\operatorname{argmax}} \prod_{k=0}^{K-1} p\left(r_{2 k} \mid \boldsymbol{r}_{2 k-2-\nu}^{2 k-1}, a_{k}, \zeta_{k}\right) \\
\cdot p\left(r_{2 k-1} \mid \boldsymbol{r}_{2 k-2-\nu}^{2 k-2}, a_{k}, \zeta_{k}\right) P\left\{a_{k}\right\}
\end{array}
$$

where $K$ is the length of the transmission and $\boldsymbol{r}_{k_{1}}^{k_{2}}$ is a shorthand notation for a vector collecting signal observations from time epoch $k_{1}$ to $k_{2}$. In the last step of (3), in order to limit the memory of the receiver, we have assumed Markovianity of order $\nu$ in the conditional observation sequence. Moreover we define a system state accounting for the 4D-TCM coder state $\mu_{k}$, the order of Markovianity $\nu$ and the ISI span $L$ as

$$
\begin{aligned}
\zeta_{k} & =\left(\mu_{k}, C_{k-1}, C_{k-2}, C_{k-3}, \ldots, C_{k-(L+\nu) / 2}\right) \\
& =\left(\mu_{k}, c_{2 k-1}, c_{2 k-2}, \ldots, c_{2 k-\nu-L}\right) .
\end{aligned}
$$

The assumed Markovianity results in an approximation whose quality increases with $\nu$.

Since we assume that the thermal and the colored noise processes have Gaussian distribution, the observation is conditionally Gaussian, given the data. The application of the chain factorization rule allows us to factor the conditional PDF in (3) as a product of two complex conditional Gaussian PDFs, completely defined by the conditional means

$$
\begin{aligned}
\hat{r}_{2 k} & =E\left\{r_{2 k} \mid \boldsymbol{r}_{2 k-2-\nu}^{2 k-1} ; a_{k}, \zeta_{k}\right\} \\
\hat{r}_{2 k-1} & =E\left\{r_{2 k-1} \mid \boldsymbol{r}_{2 k-2-\nu}^{2 k-2} ; a_{k}, \zeta_{k}\right\}
\end{aligned}
$$

and the conditional variances

$$
\begin{aligned}
\hat{\sigma}_{r_{2 k}}^{2} & =E\left\{\left|r_{2 k}-\hat{r}_{2 k}\right|^{2} \mid \boldsymbol{r}_{2 k-2-\nu}^{2 k-1} ; a_{k}, \zeta_{k}\right\} \\
\hat{\sigma}_{r_{2 k-1}}^{2} & =E\left\{\left|r_{2 k-1}-\hat{r}_{2 k-1}\right|^{2} \mid \boldsymbol{r}_{2 k-2-\nu}^{2 k-2} ; a_{k}, \zeta_{k}\right\}
\end{aligned}
$$

where $\hat{r}_{2 k}$ and $\hat{r}_{2 k-1}$ can be interpreted as linear predictive estimate of $r_{2 k}$ and $r_{2 k-1}$, respectively, and $\hat{\sigma}_{r_{2 k}}^{2}$ and $\hat{\sigma}_{r_{2 k-1}}^{2}$ as the relevant Minimum Mean Square Prediction Errors (MMSPEs) [17].The solution of a Wiener-Hopf matrix equation for linear prediction based on a 4D observable will be presented in Section III.

The detection strategy (2), the factorization (3) and linear prediction allow us to derive the branch metrics to be used for joint sequence detection and decoding in a Viterbi algorithm. Taking the logarithm, assuming that the information symbols are independent and identically distributed and discarding irrelevant terms, we can express the metric of branch $\left(a_{k}, \zeta_{k}\right)$ as

$$
\lambda_{k}\left(a_{k}, \zeta_{k}\right) \propto \sum_{i=0}^{1} \ln p\left(r_{2 k-i} \mid \boldsymbol{r}_{2 k-2-\nu}^{2 k-1-i} ; a_{k}, \zeta_{k}\right) .
$$

The detection strategy (2) can be now formalized as

$$
\hat{\boldsymbol{a}}=\underset{\boldsymbol{a}}{\operatorname{argmin}} \sum_{k=0}^{K-1} \lambda_{k}\left(a_{k}, \zeta_{k}\right)
$$

where the branch metrics are expressed as

$$
\lambda_{k}\left(a_{k}, \zeta_{k}\right)=\sum_{i=0}^{1}\left\{\frac{\left|r_{2 k-i}-\hat{r}_{2 k-i}\right|^{2}}{\hat{\sigma}_{r_{2 k-i}}^{2}}+\ln \hat{\sigma}_{r_{2 k-i}}^{2}\right\} \text {. }
$$


Finally, the state-complexity of a linear predictive receiver can be limited by means of state-reduction techniques [8][11]. Let $S=S_{c} M^{(\nu+L) / 2}$ denote the state complexity of the proposed receiver, where $S_{c}$ is the number of states of the $4 \mathrm{D}$ TCM encoder, $M$ is the cardinality of the multidimensional 4D constellation and $Q<(\nu+L) / 2+1$ denotes the memory parameter to be taken into account in the definition of a "reduced" trellis state

$$
\omega_{k}=\left(\mu_{k}, I_{k-1}(1), I_{k-2}(2), \ldots, I_{k-Q}(Q)\right)
$$

in which, for $i=1, \ldots, Q, I_{k-i}(i) \in \Omega(i)$ are subsets of the code constellation and $\Omega(i)$ are partitions of the code constellation. ${ }^{1}$ Defining $J_{i}=\operatorname{card}\{\Omega(i)\}, i=1, \ldots, Q$ as the cardinality of the partition $\Omega(i)$, the number of reduced states in the trellis diagram can be expressed as [8], [10]

$$
S^{\prime}=S_{c} \prod_{i=1}^{Q} \frac{J_{i}}{2}
$$

The branch metric can be obtained by defining a "pseudostate" [12]

$$
\left.\begin{array}{rl}
\tilde{\zeta}_{k}\left(\omega_{k}\right)= & (\underbrace{\mu_{k}, \tilde{C}_{k-1}\left(\omega_{k}\right), \ldots, \tilde{C}_{k-Q}\left(\omega_{k}\right)}_{Q+1 \text { elements }}, \\
\underbrace{\breve{C}_{k-Q-1}\left(\omega_{k}\right), \ldots, \breve{C}_{k-Q-P}\left(\omega_{k}\right)}_{P \text { code symbols }}
\end{array}\right)
$$

where $\tilde{C}_{k-1}\left(\omega_{k}\right), \ldots, \tilde{C}_{k-Q}\left(\omega_{k}\right)$ are $Q$ code symbols compatible with state $\omega_{k}$ to be found in the survivor history of state $\omega_{k}$, and $P$ are code symbols chosen by a Per-Survivor Processing (PSP) technique [11], i.e., $\breve{C}_{k-Q-1}\left(\omega_{k}\right), \ldots, \breve{C}_{k-Q-P}\left(\omega_{k}\right)$ are the $P$ 4D-TCM code symbols associated with the survivor of $\omega_{k}$. The branch metric $\tilde{\lambda}_{k}\left(I_{k}(1), \omega_{k}\right)$ in the reduced-state trellis can be defined in terms of the pseudo-state (6) according to

$$
\tilde{\lambda}_{k}\left(I_{k}(1), \omega_{k}\right)=\min _{C_{k} \in I_{k}(1)} \lambda_{k}\left(a_{k}, \tilde{\zeta}_{k}\left(\omega_{k}\right)\right)
$$

assuming that the pseudo state $\tilde{\zeta}_{k}\left(\omega_{k}\right)$ is compatible with $\omega_{k}$, i.e., $C_{k-i} \in I_{k-i}(i)$.

In the next sections, we will focus our investigation on a particular kind of colored noise, known as corona noise, and apply the proposed receiver to a high-voltage state-of-the-art power line communication system.

\section{B. Practical Implementation}

Since the optimal front-end may be quite complex from a practical point of view, requiring adaptivity and highcomputational load during the filtering process, in Fig. 2, a practical alternative is also presented. Instead of performing the whitening operation in the analog front-end stage, we propose a linear predictive receiver in which signal processing, necessary for coping with the colored noise, is entirely done in a digital fashion, i.e., modifying the branch metric of a Viterbi

\footnotetext{
${ }^{1} C_{k-i} \in \Omega(i)$ are $4 \mathrm{D}$ coded symbols compatible with the given state.
}

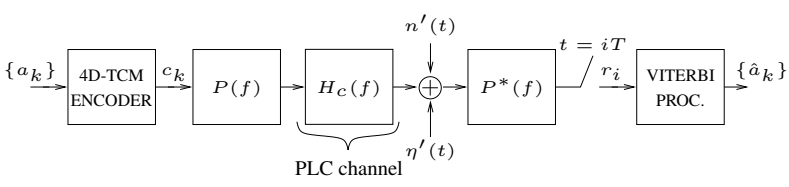

Fig. 2. Simplified system model with a practical implementation of the front-end filter.

processor. The shaping and matched filter can be both selected with square-root raised cosine frequency response, so that ISI is absent when the PLC channel is well behaved and noise samples are white when the overall noise process is white.

The proposed sub-optimal front-end allows to upgrade a state-of-the-art PLC system not designed for a scenario limited by colored noise, by simply modifying the Viterbi processor while leaving unchanged the, possibly analog, front-end stage. As previously outlined, the Viterbi processor enables sequence detection and decoding, searching an extended trellis diagram including ISI and the code memory and using a branch metric defined as in (4) and state-reduction techniques as presented in (6) and (7).

\section{Multidimensional Linear Prediction}

In this section, we describe how linear prediction can be applied to a 4D observation vector collecting $\left\{R_{k}\right\}$ and how to obtain an estimate of the colored noise samples at the output of the matched filter. We start defining a cost function $J$ which represents the mean square error between the colored noise samples and a possible set of estimates of the noise process.

It is possible to express the cost function as ${ }^{2}$

$$
\begin{aligned}
J(\boldsymbol{P})= & E\left\{\|\left[R_{k}-S_{k}\left(\boldsymbol{C}_{k-L / 2}^{k}\right)\right]\right. \\
& \left.-\sum_{i}^{\nu / 2} \boldsymbol{P}_{i}\left[\boldsymbol{R}_{k-i}-\boldsymbol{S}_{k-i}\left(\boldsymbol{C}_{k-i-L / 2}^{k-i}\right)\right] \|^{2} \mid a_{k}, \zeta_{k}\right\}
\end{aligned}
$$

where $\boldsymbol{P}$ is a matrix collecting all prediction coefficients, $S_{k}\left(\boldsymbol{C}_{k-L / 2}^{k}\right)$ is the noiseless 4D signal component affected by ISI and $\|\cdot\|^{2}$ is the Euclidean norm. The quantity $R_{k}-S_{k}\left(\boldsymbol{C}_{k-L / 2}^{k}\right)$ denotes the corona noise samples, affected by thermal noise, we want to predict, and the quantities $\left\{\boldsymbol{R}_{k-i}-\boldsymbol{S}_{k-i}\left(\boldsymbol{C}_{k-i-L / 2}^{k-i}\right)\right\}_{i}^{\nu / 2}$ represent the data [17] (i.e., the past samples of thermal and corona noise) to be used to perform linear prediction.

The cost function (8) can be expressed explicitly as

$$
\begin{aligned}
& J(\boldsymbol{P})=E\left\{\mid\left[r_{2 k-1}-s_{2 k-1}\left(\boldsymbol{c}_{2 k-1-L}^{2 k-1}\right)\right]\right. \\
& -\left.\sum_{i=1}^{\nu} p_{1, i}\left[r_{2 k-1-i}-s_{2 k-1-i}\left(\boldsymbol{c}_{2 k-1-i-L}^{2 k-1-i}\right)\right]\right|^{2} \\
& +\mid\left[r_{2 k}-s_{2 k}\left(\boldsymbol{c}_{2 k-L}^{2 k}\right)\right] \\
& \left.\quad-\left.\sum_{i=0}^{\nu} p_{2, i}\left[r_{2 k-1-i}-s_{2 k-1-i}\left(\boldsymbol{c}_{2 k-1-i-L}^{2 k-1-i}\right)\right]\right|^{2} \mid a_{k}, \zeta_{k}\right\} .
\end{aligned}
$$

\footnotetext{
${ }^{2}$ For clarity, we omit the dependence of the code symbol from the state $\zeta_{k}$ and input symbols $a_{k}$, i.e., $C_{k}\left(a_{k}, \zeta_{k}\right)=C_{k}$.
} 
Since the cost function is a sum of two positive functions of disjoint sets of variables, i.e., $J(\boldsymbol{P})=J\left(\boldsymbol{p}_{1}\right)+J\left(\boldsymbol{p}_{2}\right)$, the minimization can be performed separately on each function. In the following, we show how to obtain the prediction coefficients for the first 2D component of the 4D observable (i.e., $\left.\left\{p_{1, i}\right\}\right)$. Defining a prediction vector $\boldsymbol{p}_{1}$ for the first $2 \mathrm{D}$ observable and a data vector

$$
\boldsymbol{d}_{2 k-2-\nu}^{2 k-2}=\left(\boldsymbol{r}_{2 k-2-\nu}^{2 k-2}-\boldsymbol{s}_{2 k-2-\nu}^{2 k-2}\left(\boldsymbol{c}_{2 k-2-\nu-L}^{2 k-2}\right)\right)^{T}
$$

where $\boldsymbol{d}_{2 k-\nu}^{2 k-2}$ are $\nu$ noise samples at the output of the matched filter, we can express the cost function as ${ }^{3}$

$$
\begin{aligned}
J\left(\boldsymbol{p}_{1}\right)=E\{[ & \left.d_{2 k-1}-\boldsymbol{p}_{1}^{T} \cdot \boldsymbol{d}_{2 k-2-\nu}^{2 k-2}\right] \\
& \left.\cdot\left[d_{2 k-1}-\left(\boldsymbol{p}_{1}^{T} \cdot \boldsymbol{d}_{2 k-2-\nu}^{2 k-2}\right)^{H}\right] \mid a_{k}, \zeta_{k}\right\} .
\end{aligned}
$$

Taking the gradient with respect to the prediction vector $\boldsymbol{p}_{1}$ we are now able to formulate the Wiener-Hopf equation as

$$
\boldsymbol{R}_{\nu} \cdot \boldsymbol{p}_{1}=\boldsymbol{q}_{\nu}
$$

where the system matrix, with dimension $\nu \times \nu$, is defined as

$$
\boldsymbol{R}_{\nu}=E\left\{\left[\boldsymbol{d}_{2 k-2-\nu}^{2 k-2}\right] \cdot\left[\boldsymbol{d}_{2 k-2-\nu}^{2 k-2}\right]^{H} \mid a_{k}, \zeta_{k}\right\}
$$

and the vector of $\nu$ known terms is

$$
\boldsymbol{q}_{\nu}=E\left\{d_{2 k-1} \boldsymbol{d}_{2 k-2-\nu}^{2 k-2} \mid a_{k}, \zeta_{k}\right\} .
$$

We remark that the noise samples $\boldsymbol{d}_{2 k-2-\nu}^{2 k-2}$ are not available at the detector: they must be evaluated through the observation of the output of the front-end and a reconstruction of noiseless signal components associated with the survivor path leading to state $\zeta_{k}$.

The linear system defined in (9) can now be solved using Cholesky factorization [17], obtaining the prediction coefficient vector

$$
\boldsymbol{p}_{1}=\boldsymbol{R}_{\nu}^{-1} \cdot \boldsymbol{q}_{\nu} .
$$

As to the second 2D observable, the prediction coefficients $\left\{p_{2, i}\right\}$ and the cost function $J\left(\boldsymbol{p}_{2}\right)$ can be determined in a similar manner, noting that in the evaluation of the estimate $E\left\{r_{2 k} \mid \boldsymbol{r}_{2 k-2-\nu}^{2 k-1} ; a_{k}, \zeta_{k}\right\}$ we can also use the observable at time $2 k-1$ from the first $2 \mathrm{D}$ code symbol.

Finally, rewriting the cost functions $J\left(\boldsymbol{p}_{1}\right)$ and $J\left(\boldsymbol{p}_{2}\right)$ as explicit functions of the predictor vectors $\boldsymbol{p}_{1}$ and $\boldsymbol{p}_{2}$, respectively, we can express the Minimum Mean Square Prediction Errors as

$$
\begin{aligned}
& J\left(\boldsymbol{p}_{1}\right)=\sigma_{\eta}^{2}+\sigma_{n}^{2}-\boldsymbol{p}_{1}^{T} \cdot \boldsymbol{q}_{\nu} \\
& J\left(\boldsymbol{p}_{2}\right)=\sigma_{\eta}^{2}+\sigma_{n}^{2}-\boldsymbol{p}_{2}^{T} \cdot \boldsymbol{q}_{\nu+1}
\end{aligned}
$$

where $\sigma_{n}^{2}$ is the colored noise power and $\sigma_{\eta}^{2}$ the thermal noise power at the input of the Viterbi processor.

\footnotetext{
${ }^{3}$ Superscripts $T$ and $H$ denote transpose and Hermitian transpose operators.
}

\section{Corona Noise Model}

Corona noise is a predominant noise source which characterizes high-voltage power line channels in normal operation. The PLC channel may consist of one or more conductors, depending on the considered coupling scheme, i.e., phase-toground or phase-to-phase [18]. Corona noise is a common noise source for high-voltage transmission lines, since it is permanent and depends on (i) the service voltage, (ii) the geometric configuration of the power line, (iii) the type of conductors involved in the line and (iv) the atmospheric conditions.

Corona noise is caused by partial discharges on insulators and in air surrounding electrical conductors of power lines. When high-voltage power lines are in operation, the power frequency voltage originates a strong electric field in the vicinity of the conductor. This electric field accelerates free electrons present in the air nearby conductors: these electrons collide with molecules of the air, generating a free electron and positive ion couple. This process continues forming an avalanche phenomenon called "corona discharge". The positive and negative charges motion induces a current both in the conductors and ground.

The induced current appears like a train of current pulses, with random pulse amplitude variations and random interarrival intervals. The injected current due to corona noise on one conductor can be modeled by a current source [4], [19]: according to Shockley-Ramo theorem [18], a corona discharge induces current in all conductors, i.e., each conductor of the power line channel is connected to the ground by a current source.

A few corona noise models are presented in the literature [1], [4]-[6]: in this article, the model proposed in [5], [6] is considered. Corona noise, as a random signal, is characterized equivalently through its autocorrelation function or its frequency spectrum. To this purpose, the corona noise spectrum is generated by a method that takes into account the generation phenomena of corona currents injected in the conductors and the propagation along the line [20], [21]. This spectrum is utilized to synthesize an autoregressive (AR) digital filter [17], whose output is described by the expression

$$
n_{k}=\sum_{\ell=1}^{N} f_{\ell} n_{k-\ell}+w_{k}
$$

where $\left\{w_{k}\right\}$ is a sequence of independent zero-mean Gaussian random variables and $\left\{f_{\ell}\right\}_{\ell=1}^{N}$ is the set of coefficients modeling the corona noise process. The synthesis of the digital filter essentially calls for the identification of the coefficients $\left\{f_{\ell}\right\}_{\ell=1}^{N}$ and can be done using a procedure based on the maximum entropy method proposed in [22] or on the minimization of the difference between the estimated frequency spectrum and the measured power spectrum. Tab. I shows a complete set of coefficients modeling the corona noise for different voltage lines with carrier couplings of lateral phase-to-ground type [6].

Note that (10) defines a corona power spectrum whose frequency components are over the entire frequency domain, 


\begin{tabular}{|c|c|c|c|c|}
\hline Voltage $[\mathrm{kV}]$ & $f_{1}$ & $f_{2}$ & $f_{3}$ & $f_{4}$ \\
\hline \hline 225 & -1.225 & 1.052 & -0.603 & 0.217 \\
\hline 380 & -1.298 & 1.109 & -0.625 & 0.210 \\
\hline 750 & -1.302 & 1.041 & -0.611 & 0.207 \\
\hline 1050 & -1.292 & 1.080 & -0.647 & 0.224 \\
\hline
\end{tabular}

TABLE I

VALUES OF THE DIGITAL FILTER COEFFICIENTS $\left\{f_{\ell}\right\}_{\ell=1}^{4}$.

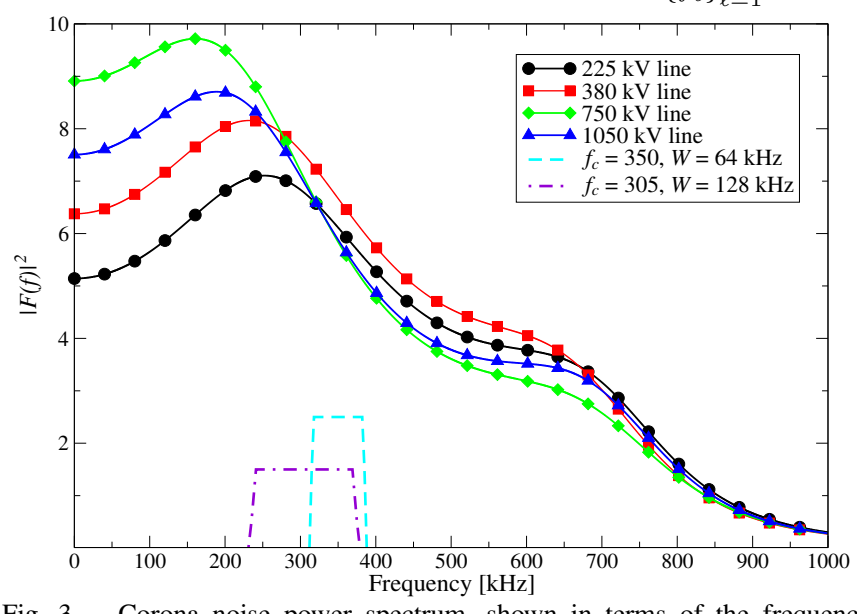

Fig. 3. Corona noise power spectrum, shown in terms of the frequency response $F(f)$ of the AR filter in (10).

i.e., its bandwidth is generally greater than that used by the transmission system. In our simulation, we derive an equivalent complex low pass filtered version of the corona noise process within the bandwidth of the considered signaling scheme. The filter used for the generation of corona noise is a finite impulse response (FIR) complex filter with coefficients obtained using Cholesky factorization [17] applied to the complex low pass filtered corona noise power spectrum. Finally, in Fig. 3, the corona noise power spectrum obtained with the AR model presented in (10) with coefficient shown in Tab. I, along with the frequency carriers $f_{c}$ and the bandwidths $W$ used by the systems considered in our simulations are also shown.

\section{Numerical Results}

Since in this paper we are only dealing with the effects of colored noise sources on the performance of single carrier systems, we assume that the channel frequency response over the used transmission bandwidth can be considered flat or approximately flat to enable a correct equalization of the received signal. As a consequence, we assume, from now on, $L=0$ and introduce a parameter called the Corona to Thermal noise Ratio (CTR), defined as CTR $=\sigma_{n}^{2} / \sigma_{\eta}^{2}$.

The signal-to-noise ratio (SNR) is defined as $E_{b} / N_{0}$, where $E_{b}$ is the received energy per information bit, and $N_{0}$ is the white noise monolateral power spectral density when corona noise is absent. In the presence of corona noise, $N_{0}$ is defined as the equivalent white noise intensity which yields the total noise variance $\sigma^{2}=\sigma_{\eta}^{2}+\sigma_{n}^{2}=\sigma_{\eta}^{2}(1+\mathrm{CTR})$ at the input of the Viterbi processor.

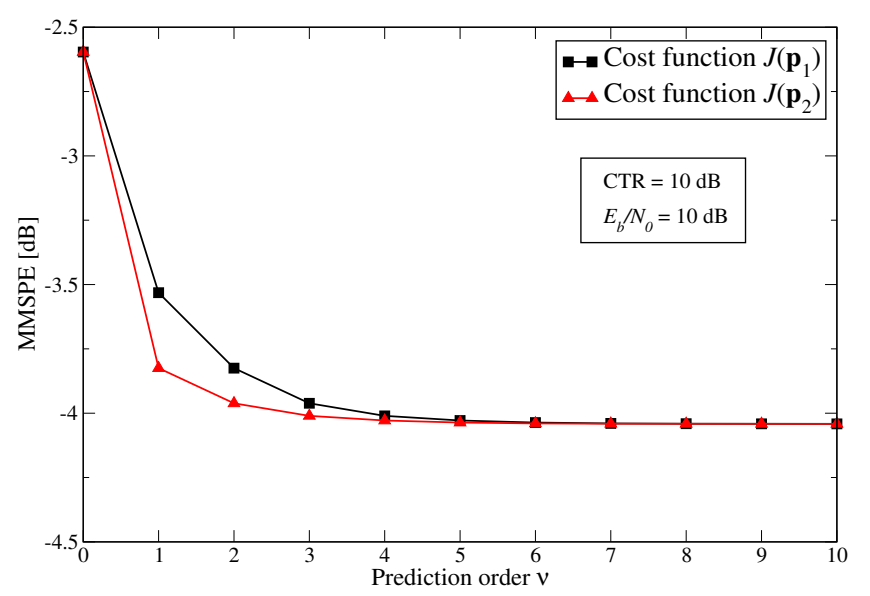

Fig. 4. MMSPEs, normalized to the power of the signal $s_{i}\left(\boldsymbol{c}_{i-L}^{i}\right)$, as a function of the prediction order $\nu$.

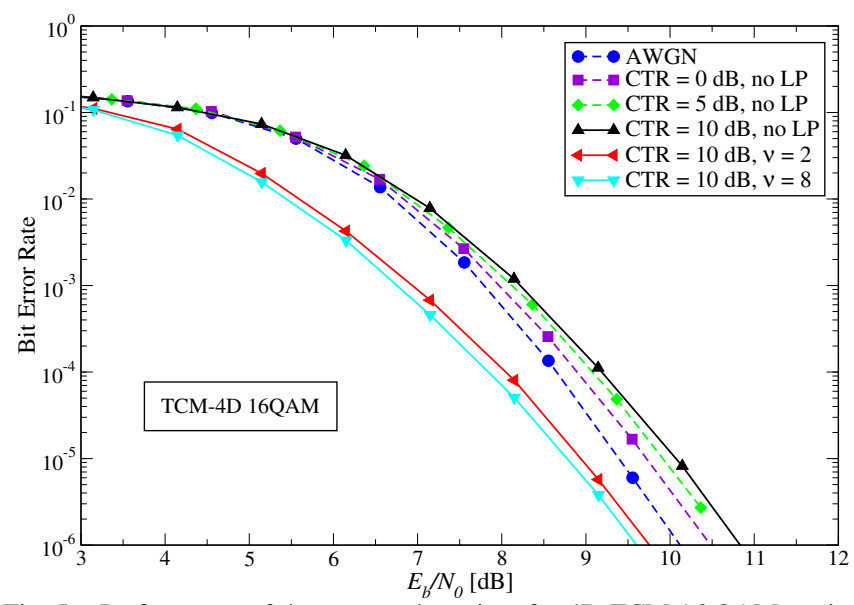

Fig. 5. Performance of the proposed receiver for 4D-TCM 16 QAM, various values of CTR and prediction order.

\section{A. MMSPE analysis}

In Fig. 4 the performance of the linear estimator is assessed in terms of MMSPEs versus the prediction order $\nu$ for a fixed $E_{b} / N_{0}$ of $10 \mathrm{~dB}$. The corona noise process is generated according to the model for a $380 \mathrm{kV}$ line in Tab. I with signaling bandwidth equal to $W=64 \mathrm{kHz}$ centered around $f_{c}=350 \mathrm{kHz}$ and $16 \mathrm{QAM}$ constellation. Note that the prediction order $\nu$ is referred to a $2 \mathrm{D}$ code symbol time, i.e., $\nu=2$ means that two $2 \mathrm{D}$ observables are needed for the computation of $\hat{r}_{2 k-1}$. The gain shown in Fig. 4 is approximately $1.4 \mathrm{~dB}$ for a CTR $=10 \mathrm{~dB}$.

\section{B. BER analysis}

Dashed lines in Fig. 5 show the penalty, due to corona noise, in terms of degradation of $E_{b} / N_{0}$, of a single carrier commercial PLC system using an 8-state 4D-TCM code with 16 QAM constellation without linear prediction, for different values of CTR. We assume the $380 \mathrm{kV}$ line in Tab. I with channel frequency response presented in [23], a transmit bandwidth and a carrier frequency equal to, respectively, $W=64 \mathrm{kHz}$ and $f_{c}=350 \mathrm{kHz}$. In Fig. 5 we also show the performance, in terms of $E_{b} / N_{0}$, of the proposed simplified linear predictive receiver. The curve obtained without linear prediction ("no 


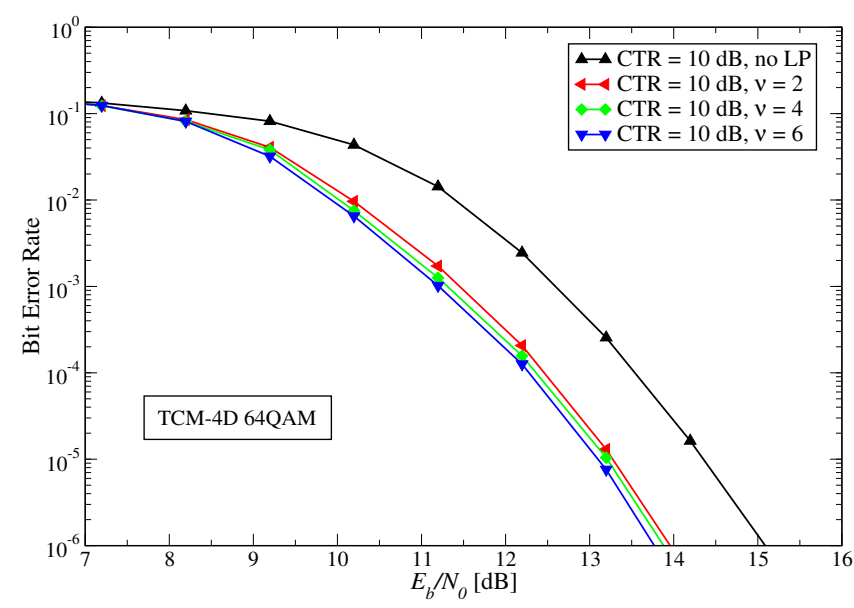

Fig. 6. Performance of the proposed receiver for 4D-TCM 64 QAM and various values of prediction order.

LP" curve) with CTR $=10 \mathrm{~dB}$ is the performance of a stateof-the-art single carrier system which operates with a trellis complexity of $S=8$ entirely implementable on a single digital signal processor (DSP). The BER curves in Fig. 5 were obtained using a reduced state defined as $\omega_{k}=\mu_{k}$, i.e., including only the state of the TCM coder $(Q=0)$, and extracting the past $\nu / 2$ 4D-TCM code symbols using a PSP approach ( $P$ equal to half the prediction order $\nu$ ). This set of state parameters allows one to implement a Viterbi algorithm, according to (5), with a number of reduced states equal to $S^{\prime}=8$, which does not increase the computational load on the DSP. Note that with only a prediction order $\nu=2$ it is possible to obtain almost all the SNR gain achievable using linear prediction, in perfect agreement with the MMSPE results presented in Fig. 4.

Fig. 6 shows the improvement, in terms of $E_{b} / N_{0}$ obtainable using the proposed simplified linear predictive receiver considering a 64 QAM constellation scheme operating over the $1050 \mathrm{kV}$ line in Tab. I, with signal bandwidth equal to $W=128 \mathrm{kHz}$ and carrier frequency $f_{c}=305 \mathrm{kHz}$. The BER curves in Fig. 6 were obtained using different values of the prediction order $\nu$, a reduced state defined as $\omega_{k}=$ $\left(\mu_{k}, I_{k-1}(1)\right)$, i.e., $Q=1$ with $J_{1}=8$, and extracting the past $\nu$ 2D code symbols using PSP ( $P$ equal to half the prediction order $\nu$ ). This set of state parameters allows one to use a Viterbi processor searching a trellis diagram, according to (5), with a reduced number of states equal to $S^{\prime}=32$, which may still be a tolerable load for a DSP.

\section{CONCLUSIONS}

In this paper, optimal and practical receivers based on linear prediction and reduced-state sequence detection applied to single carrier PLC system operating on a channel limited by colored Gaussian noise are presented. The proposed solution may be able to improve the $E_{b} / N_{0}$ performance of a conventional state-of-the-art single carrier system and make the receiver more robust against colored noise. As a case study, the proposed receiver was shown to be effectively applicable to a high-voltage PLC channel limited by corona noise.

\section{ACKNOWLEDGMENTS}

This work was supported by Selta S.p.A., Roveleto di Cadeo (Piacenza), Italy.

\section{REFERENCES}

[1] A. Mujcić, N. Suljanović, M. Zajc, and J. F. Tasic, "Power line noise model appropriate for investigation of channel coding methods," in Eurecon 2003, Ljubljana, Slovenia, June 2003, pp. 299-303.

[2] H. Meng, Y. L. Guan, and S. Chen, "Modeling and analysis of noise effects on broadband power-line communications," IEEE Trans. Power Delivery, vol. 20, no. 2, pp. 630-637, April 2005.

[3] M. Zimmermann and K. Dostert, "Analysis and modeling of impulsive noise in broadband power-line communications," IEEE Trans. Electromagnetic Compatibility, vol. 44, no. 1, pp. 249-258, February 2002.

[4] N. Suljanović, A. Mujcić, M. Zajc, and J. F. Tasic, "Computation of high-frequency and time characteristics of corona noise on $\mathrm{HV}$ power line," IEEE Trans. Power Delivery, vol. 10, no. 3, pp. 71-79, July 2005.

[5] P. Burrascano, S. Cristina, and M. D'Amore, "Performance evaluation of digital signal transmission channels on coronating power lines," in ISCAS'88, 1988, pp. 365-368.

[6] - "Digital generator of corona noise on power line carrier channels," IEEE Trans. Power Delivery, vol. 3, no. 3, pp. 850-856, July 1988.

[7] E. Eleftheriou and W. Hirt, "Improving performance of PRML/EPRML through noise prediction," IEEE Trans. Magnetics, vol. 32, no. 5, pp. 3968-3970, September 1996.

[8] M. V. Eyuboglu and S. U. H. Qureshi, "Reduced-state sequence estimation with set partitioning and decision feedback," IEEE Trans. Commun., vol. 36, no. 1, pp. 13-20, January 1988.

[9] A. Duel-Hallen and C. Heegard, "Delayed decision-feedback sequence estimation," IEEE Trans. Commun., vol. 37, no. 5, pp. 428-436, May 1989.

[10] P. R. Chevillat and E. Eleftheriou, "Decoding of trellis-encoded signals in the presence of intersymbol interference and noise," IEEE Trans. Commun., vol. 37, no. 7, pp. 669-676, July 1989.

[11] R. Raheli, A. Polydoros, and C. K. Tzou, "Per-Survivor Processing: a general approach to MLSE in uncertain environments," IEEE Trans. Commun., vol. 41, no. 2, pp. 354-364, February 1995.

[12] G. Ferrari, G. Colavolpe, and R. Raheli, Detection algorithms for wireless communications, with applications to wired and storage systems. London, UK: John Wiley \& Sons, 2004.

[13] L. F. Wei, "Trellis coded modulation with multidimensional constellation," IEEE Trans. Commun., vol. IT-33, no. 4, pp. 483-501, July 1987.

[14] M. K. Simon, S. M. Hinedi, and W. C. Lindsey, Digital communication techniques: signal design and detection. Englewood Cliffs, New Jersey: Prentice Hall PTR, 1994.

[15] G. Ungerboeck, "Channel coding with multilevel/phase signals," IEEE Trans. Inform. Theory, vol. 28, pp. 55-67, January 1982.

[16] G. Ferrari, G. Colavolpe, and R. Raheli, "A unified framework for finitememory detection," IEEE J. Select. Areas Commun., vol. 23, no. 9, pp. 1697 - 1706, September 2005.

[17] S. Haykin, Adaptive Filter Theory, 4th ed. New York: Prentice-Hall International Editions, 2001.

[18] P. S. Maruvada, Corona performance on High-Voltage transmission lines. Baldock, U.K.: Research Studies Press Ltd., 2000.

[19] N. Suljanović, A. Mujcić, M. Zajc, and J. F. Tasic, "Corona noise characteristics in high voltage PLC channel," in ICIT, Maribor, Slovenia, June 2003, pp. 1036-1039.

[20] S. Cristina and M. D'Amore, "Analytical method for calculating corona noise on HVAC power line carrier communications channels," IEEE Trans. on PAS, vol. PAS-104, no. 5, May 1985.

[21] P. Burrascano, S. Cristina, and M. D'Amore, "Digital generator of corona noise on power line carrier channels," in IEEE PES Summer Meeting, San Francisco, July 1987.

[22] J. P. Burg, "Maximum entropy spectral analysis," in Proc. 37-th Meeting Soc. of Explor. Geophysicists, Oklaoma City, USA, 1967.

[23] R. Pighi and R. Raheli, "On multicarrier signal transmission for highvoltage power lines," in Proc. Int. Symp. Power-Line Commun. and Its Apps., Vancouver, Canada, April 2005. 